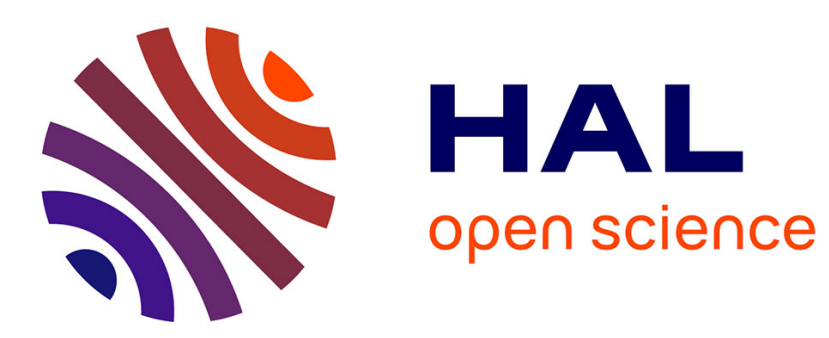

\title{
State Observability in Presence of Disturbances: the Analytic Solution and its Application in Robotics
}

\author{
Agostino Martinelli
}

\section{To cite this version:}

Agostino Martinelli. State Observability in Presence of Disturbances: the Analytic Solution and its Application in Robotics. IROS 2017 - IEEE/RSJ International Conference on Intelligent Robots and Systems, Sep 2017, Vancouver, Canada. pp.1-8. hal-01669046

\section{HAL Id: hal-01669046 \\ https://hal.inria.fr/hal-01669046}

Submitted on 20 Dec 2017

HAL is a multi-disciplinary open access archive for the deposit and dissemination of scientific research documents, whether they are published or not. The documents may come from teaching and research institutions in France or abroad, or from public or private research centers.
L'archive ouverte pluridisciplinaire HAL, est destinée au dépôt et à la diffusion de documents scientifiques de niveau recherche, publiés ou non, émanant des établissements d'enseignement et de recherche français ou étrangers, des laboratoires publics ou privés. 


\title{
State Observability in Presence of Disturbances: the Analytic Solution and its Application in Robotics
}

\author{
Agostino Martinelli
}

\begin{abstract}
This paper presents the analytic solution of a fundamental open problem in the framework of state estimation/nonlinear observability, which is the Unknown Input Observability problem (UIO problem). The problem consists in deriving the analytic criterion that allows us to automatically obtain the state observability in presence of disturbances (or unknown inputs). In other words, the problem is to extend the well known observability rank condition to the case when the dynamics are also driven by unknown inputs. Enunciated in the seventies by the control theory community, this problem was only solved in the linear case. The solution here provided holds for nonlinear systems in presence of a single unknown input. The first part of the paper presents this analytic solution. Very surprisingly, the complexity of the overall analytic criterion is comparable to the complexity of the observability rank condition. The second part of the paper applies this analytic criterion to a robotics system when its dynamics are affected by an external disturbance (e.g., due to the presence of wind). To corroborate the results of our observability analysis we perform extensive simulations and we show that, a simple estimator based on an Extended Kalman Filter, provides results that agree with what we could expect from the observability analysis.
\end{abstract}

\section{INTRODUCTION}

State observability is a necessary condition that a state must satisfy to be estimated. In robotics, where the systems can be often very complex, intuitive reasoning to obtain the observable state can fail. For, performing an observability analysis prior to solving an estimation problem can be fundamental. This has been done by many authors to solve several problems (e.g., in SLAM [4], [11], [12], [18], [29], [30], [31], [33], in visual-inertial sensor fusion [9], [10], [13], [15], [16], [17], [19], [21], [26], [28], in sensor calibration [5], [7], [27]). Investigating the observability properties is very simple in the linear case. Unfortunately, real systems are very rarely characterized by linearity.

The control theory community has introduced the analytic criterion necessary to check the state observability for nonlinear systems provided that all the system inputs are known (i.e., in absence of disturbances). This is the observability rank condition introduced by Herman and Krener in 1977 [8]. In accordance with this criterion, it is possible to obtain all the observability properties of a nonlinear system by performing automatic computation. On the other hand, in many real scenarios, one or more disturbances can dramatically impact the system dynamics. A disturbance can be considered as an unknown input (UI). Its presence can dramatically affect the observability properties of the state.

\footnotetext{
*This work was supported by the French National Research Agency ANR 2014 through the project VIMAD

A. Martinelli is with INRIA Rhone Alpes, Grenoble, France e-mail: agostino.martinelli@inria.fr
}

This is for instance the case of a drone that operates in presence of wind (or a boat that operates in presence of stream). The wind (or the stream) is in general unknown, time-variant and acts on the system dynamics as an unknown input.

The problem of finding the analytic criterion able to determine the observability properties in presence of disturbances was enunciated and firstly investigated by the automatic control community in the seventies [1], [3], [6], [32]. In particular, Basile and Marro provided the solution of this problem in the linear case [1]. In robotics, most of the systems are characterized by nonlinear dynamics, even in the very simple case of planar robotics. Additionally, the presence of disturbances cannot be ignored in many cases and can significantly alter the observability properties. For these reasons, this problem in the nonlinear case has been investigated by the robotics community and partial solutions have recently been proposed [2], [21]. These solutions are heuristic solutions to the UIO problem and only provide sufficient conditions for the state observability. They are based on a suitable state extension.

This paper provides the analytic solution of the UIO problem in the nonlinear case when the dynamics are driven by multiple known inputs and a single unknown input. A preliminary solution of this problem was presented in [23]. Here, this result is completed by providing all the convergence properties of the algorithm in [23]. Finally, the general analytic solution that holds for any nonlinear system driven by any number of disturbances is provided in [25].

In [8], [14] the observability properties of a nonlinear system are obtained by computing the observable codistribution 1 . The computation of this codistribution is the core of the observability rank condition introduced in [8]. In order to deal with the case of unknown inputs, we need to derive a new algorithm able to generate the observable codistribution. In the first part of section II we remind the reader the algorithm to compute the observable codistribution in the case without disturbances. Then, we introduce the new algorithm that generates the entire observable codistribution

\footnotetext{
${ }^{1}$ The reader non-familiar with the concept of distribution, as it is used in [14], should not be afraid by the term distribution and the term codistribution. Very simply speaking, a distribution is a vector space defined on $M$ (our set in $\mathbb{R}^{n}$ where the system is defined). In particular, this vector space changes by moving on $M$. This vector space is in fact the span of a set of vector functions (vector fields) defined on $M$. A codistribution is the dual of a distribution. Very simply speaking (and this is enough to understand the theory of nonlinear observability) a distribution is generated by a set of column vectors. A codistribution is generated by a set of line vectors. All these vectors are vector functions (i.e., they depend on the point $x \in M)$ and they have the same dimension of $x$.
} 
in presence of a single unknown input. In section II-B we provide its convergence properties, by a comparison with the case without disturbances. The solution of the UIO problem in the case of a single unknown input is summarized in section II-C. Then, the paper applies this criterion to obtain the observability properties of a robotic system. In section III we define our robotics problem. It consists of a robot that moves in $3 D$. This could be an aerial vehicle equipped with airspeed, gyroscopes and monocular vision sensors (but it could also be an underwater vehicle). We assume that the vehicle dynamics are affected by an external disturbance (e.g., due to the presence of wind). We apply the proposed analytic criterion to obtain the observability properties of this system. In section III-D we perform extensive simulations and we introduce a simple estimator. The results obtained by estimating the state with this estimator fully agree with our observability analysis. In section IV we provide our conclusion. We conclude this section by also mentioning new important analytic results recently obtained.

\section{EXTENSION OF THE OBSERVABILITY RANK CONDITION}

We refer to a nonlinear control system with $m_{u}$ known inputs $\left(u \triangleq\left[u_{1}, \cdots, u_{m_{u}}\right]^{T}\right)$ and a single unknown input or disturbance $(w)$. The state is the vector $x \in M$, with $M$ an open set of $\mathbb{R}^{n}$. We assume that the dynamics are nonlinear with respect to the state and linear with respect to the inputs (both known and unknown). Finally, for the sake of simplicity, we will refer to the case of a single output $y$ (the extension to multiple outputs is straightforward). Our system is characterized by the following equations:

$$
\left\{\begin{array}{l}
\dot{x}=\sum_{i=1}^{m_{u}} f^{i}(x) u_{i}+g(x) w \\
y=h(x)
\end{array}\right.
$$

where $f^{i}(x), i=1, \cdots, m_{u}$, and $g(x)$ are vector fields in $M$ and the function $h(x)$ is a scalar function defined on the open set $M$. We denote by $x_{0}$ the state in $M$ where we are interested in deriving the observability properties. We denote by the symbol $\nabla$ the gradient with respect to the state $x$. Additionally, for a given codistribution $\Omega$ and a given vector field $\theta$ (both defined on the open set $M$ ), we denote by $\mathcal{L}_{\theta} \Omega$ the codistribution whose covectors are the Lie derivatives along $\theta$ of the covectors in $\Omega$. Finally, given two vector spaces $V_{1}$ and $V_{2}$, we denote by $V_{1}+V_{2}$ their sum, i.e., the span of all the generators of both $V_{1}$ and $V_{2}$.

Note that this section directly provides the analytic results. All the analytic proofs can be found in [24].

\section{A. Analytic computation of the observable codistribution}

We start by reminding the reader the algorithm that computes the observable codistribution in absence of disturbances (namely, the core of the observability rank condition introduced by Herman and Krener [8]). In absence of disturbances, i.e., when also the input $w$ is a known input, the observable codistribution is generated by the following recursive algorithm (see [8] and [14]):

Algorithm 1 Observable codistribution when $w$ is known

1) $\Omega_{0}=\operatorname{span}\{\nabla h\}$;

2) $\Omega_{m}=\Omega_{m-1}+\sum_{i=1}^{m_{u}} \mathcal{L}_{f^{i}} \Omega_{m-1}+\mathcal{L}_{g} \Omega_{m-1}$

In presence of multiple outputs, we only need to add to the codistribution $\Omega_{0}$, the span of the gradients of the remaining outputs.

Let us consider now the case when the input $w$ is unknown. We will denote by $L_{g}^{1}=L_{g}^{1}(x)$ the first order Lie derivative of the function $h(x)$ along the vector field $g$, i.e.:

$$
L_{g}^{1} \triangleq \mathcal{L}_{g} h \triangleq \nabla h \cdot g(x)
$$

The analytic computation of the observable codistribution is based on the assumption that $L_{g}^{1} \neq 0$ on a given neighbourhood of $x_{0}$. If this hypothesis is not satisfied, it is possible either to redefine the output such that this assumption holds or to prove that the presence of the disturbance does not affect the state observability ${ }^{2}$ Hence, we are allowed to assume that $L_{g}^{1} \neq 0$ on a given neighbourhood of $x_{0}$. The algorithm that generates the observable codistribution is the following:

\section{Algorithm 2 Observable codistribution when $w$ is unknown \\ 1) $\Omega_{0}=\operatorname{span}\{\nabla h\}$; \\ 2) $\Omega_{m}=\Omega_{m-1}+\sum_{i=1}^{m_{u}} \mathcal{L}_{f^{i}} \Omega_{m-1}+\mathcal{L}_{\frac{g}{L_{g}^{1}}} \Omega_{m-1}+$ $\sum_{i=1}^{m_{u}} \mathcal{L}_{i_{\phi}-1} \nabla h$}

where the vectors ${ }^{i} \phi_{m} \in \mathbb{R}^{n}\left(i=1, \cdots, m_{u}\right)$ are defined by the following algorithm:

\section{Algorithm 3}

1) ${ }^{i} \phi_{0}=f^{i}$

2) ${ }^{i} \phi_{m}=\frac{\left[{ }^{i} \phi_{m-1}, g\right]}{L_{g}^{1}}$

where the parenthesis $[\cdot, \cdot]$ denote the Lie brackets of vector fields, defined as follows:

$$
[f, g] \triangleq \frac{\partial g}{\partial x} f(x)-\frac{\partial f}{\partial x} g(x)
$$

In presence of multiple outputs, we only need to add to the codistribution $\Omega_{0}$, the span of the gradients of the remaining outputs. Note that, in presence of multiple outputs, the function $L_{g}^{1}$ is still a scalar function since it is still defined by using a single output. The result is independent of the chosen output (provided that $L_{g}^{1}$ does not vanish ${ }^{3}$.

We conclude this subsection by remarking the differences between algorithm 1 and 2 . They are:

- In the recursive step, the term $\mathcal{L}_{g} \Omega_{m-1}$ must be replaced by $\mathcal{L}_{\frac{g}{L_{g}^{1}}} \Omega_{m-1}$. In other words, the vector field that corresponds to the unknown input (i.e., $g$ ) must be rescaled by dividing by $L_{g}^{1}$.

\footnotetext{
${ }^{2}$ The reader is addressed to [24] to see the definition of the new output.

${ }^{3}$ The case when $L_{g}^{1}=0$ for all the outputs is dealt in [24].
} 
- The recursive step also contains the sum of the contributions $\sum_{i=1}^{m_{u}} \mathcal{L}_{{ }^{i} \phi_{m-1}} \nabla h$. In other words, we need to compute the Lie derivatives of the gradient of the output $h(x)$ along the vector fields obtained through the recursive algorithm 3 .

\section{B. Convergence properties of algorithm 2}

We start by summarizing the convergence properties of algorithm 1] In [14] it is proven that this algorithm converges in an open and dense set of $M$. In particular, from lemmas 1.9.1, 1.9.2 and 1.9.6 in [14], we know that the convergent codistribution is obtained at the smallest integer $m$ for which $\Omega_{m}=\Omega_{m+1}$ and $m \leq n-1$. This analytic result is a consequence of the fact that, all the terms that appear at the recursive step of algorithm 1 , are the Lie derivative of the codistribution at the previous step, along fixed vector fields (i.e., vector fields that remain the same at each step of the algorithm).

Let us consider now the recursive step of algorithm 2. As mentioned above, the first difference with the recursive step of algorithm 1 , is the term $\mathcal{L}_{\frac{g}{L_{g}^{1}}} \Omega_{m-1}$, which replaces the term $\mathcal{L}_{g} \Omega_{m-1}$. However, this new term is still the Lie derivative of the codistribution at the previous step along the fixed vector field $\frac{g}{L_{g}^{1}}$. Hence, the presence of this term, does not alter the convergence properties with respect to the ones that hold for algorithm 1 . On the other hand, the nature of the last term at the recursive step of algorithm 2 (i.e., $\left.\sum_{i=1}^{m_{u}} \mathcal{L}_{i} \phi_{m-1} \nabla h\right)$ significantly differs from all the other terms. Indeed, the vector fields along with we are computing the Lie derivatives (i.e., ${ }^{i} \phi_{m-1}$ ) change at each step $m$ of algorithm 2. In other words, they are not fixed vector fields. Additionally, the Lie derivative is not computed on the codistribution at the previous step of the algorithm but directly on the gradient of the output $(\nabla h)$.

Therefore, we distinguish the case when the contribution of this term is included in the other terms from the case when it is not included. In the former case, the convergence criterion of algorithm 2 would simply consist of the inspection of the equality $\Omega_{m+1}=\Omega_{m}$, as for algorithm 1 and we conclude that, in this case, the convergence of algorithm 2 is attained in at most $n-1$ step 4

The following result provides a simple criterion to check, by a finite number of operations, if a given system belongs to this former case. Without loss of generality, we consider the case when $m_{u}=1$ (we denote by $\phi_{m} \triangleq 1 \phi_{m}$ ). We have the following result:

Lemma 1 Let us denote by $\Lambda_{j}$ the distribution generated by $\phi_{0}, \phi_{1}, \cdots, \phi_{j}$ (i.e., the vectors obtained by running algorithm 3 ) and by $m(\leq n-1)$ the smallest integer for which $\Lambda_{m+1}=\Lambda_{m}$ ( $n$ is the dimension of the state $x$ ). If $\mathcal{L}_{\phi_{j}} L_{g}^{1}=0, \forall j=0, \cdots, m$, the convergence of algorithm 2 occurs in at most $n-1$ steps and it occurs at the smallest integer $j$ such that $\Omega_{j+1}=\Omega_{j}$.

${ }^{4}$ In other words, it is possible to repeat all the steps of lemmas 1.9.1, 1.9.2 and 1.9.6 in [14], for algorithm 2 without the term $\sum_{i=1}^{m_{u}} \mathcal{L}_{i} \phi_{m-1} \nabla h$.
Proof: See the proof of lemma 6 in [24]

Note that the existence of an integer $m(\leq n-1)$ such that $\Lambda_{m+1}=\Lambda_{m}$ is proved in [14]. In particular, in the first chapter of [14], lemmas 1.8 .2 and 1.8.3 analyze the convergence of $\Lambda_{j}$ with respect to $j$. Note that our case corresponds to the case analyzed in [14] when $\Delta$ is the span of $f$, and when $\tau_{1}, \cdots, \tau_{q}$ are the single vector field $g$. Additionally, in [14] there is not the term $L_{g}^{1}$. Namely, our case corresponds to the case analyzed in [14], when $L_{g}^{1}=1$. On the other hand, it is immediate to repeat all the steps in lemmas 1.8.2 and 1.8.3 of [14] with a general expression of $L_{g}^{1}$ and prove the following result: The distribution $\Lambda_{j}$ converges to $\Lambda^{*}$ on an open and dense set in $M$ and the convergence is achieved at the smallest integer for which we have $\Lambda_{m+1}=\Lambda_{m}$. Additionally, we have $\Lambda_{m+1}=\Lambda_{m}=\Lambda^{*}$ and $m$ cannot exceed $n-1$.

Let us derive an upper bound for the number of computations necessary to check if the assumptions of lemma 1 are met. We need to check that $\mathcal{L}_{\phi_{j}} L_{g}^{1}=0, \forall j=0, \cdots, m$, where $m$ is the smallest integer for which $\Lambda_{m+1}=\Lambda_{m}$. Hence, we need to run at most the first $n-1$ steps of algorithm 3 to obtain $\phi_{0}, \phi_{1}, \cdots, \phi_{n-1}$ and compute at most $n$ Lie derivatives of $L_{g}^{1}$, i.e., $\mathcal{L}_{\phi_{0}} L_{g}^{1}, \mathcal{L}_{\phi_{1}} L_{g}^{1}, \cdots, \mathcal{L}_{\phi_{n-1}} L_{g}^{1}$. Therefore, we have:

Remark 1 Checking that the assumptions of Lemma 1] are met, only requires to perform a finite number of (simple) operations

Let us consider now the case when the assumptions of Lemma 1 are not met. This is the most frequent case. The criterion needs to compute the following key quantity:

$$
\tau \triangleq \frac{\mathcal{L}_{g}^{2} h}{\left(L_{g}^{1}\right)^{2}}
$$

When the assumptions of Lemma 1 are not satisfied, it is possible to prove that it exists $m^{\prime}$ such that $\nabla \tau \in \Omega_{m^{\prime}}$ (and therefore $\nabla \tau \in \Omega_{m} \forall m \geq m^{\prime}$ ). Additionally, the convergence of algorithm 2 has been reached when $\Omega_{m+1}=$ $\Omega_{m}$, and $m \geq m^{\prime}$. Finally, in this case, the convergence is attained in at most $n+2$ step 5

\section{Summary of the analytic criterion}

We conclude this section by outlining the steps to investigate the weak local observability at a given point $x_{0}$ of a nonlinear system driven by a single disturbance and several known inputs. In other words, to investigate the weak local observability of a system defined by a state that satisfies (1). Basically, these steps are the steps necessary to compute the observable codistribution (i.e., the steps of algorithms 2 and 3 and to prove that the gradient of a given state component belongs to this codistribution.

1) For the chosen $x_{0}$, compute $L_{g}^{1}\left(=\mathcal{L}_{g}^{1} h\right)$. In the case when $L_{g}^{1}=0$ redefine the output, as explained in[24].

\footnotetext{
${ }^{5}$ The reader is addressed to [24] where the proofs of all these statements are provided.
} 
2) Compute the codistribution $\Omega_{0}$ and $\Omega_{1}$ (at $x_{0}$ ) by using algorithm 2

3) Compute the vector fields ${ }^{i} \phi_{m}\left(i=1, \cdots, m_{u}\right)$ by using algorithm 3, starting from $m=0$, to check if the considered system is in the case dealt by Lemma 1. In this case, set $m^{\prime}=0$, use the recursive step of algorithm 2 to build the codistribution $\Omega_{m}$ for $m \geq 2$, and skip to step 6.

4) Compute $\tau\left(=\frac{\mathcal{L}_{g}^{2} h}{\left(L_{g}^{1}\right)^{2}}\right)$ and $\nabla \tau$.

5) Use the recursive step of algorithm 2 to build the codistribution $\Omega_{m}$ for $m \geq 2$, and, for each $m$, check if $\nabla \tau \in \Omega_{m}$. Denote by $m^{\prime}$ the smallest $m$ such that $\nabla \tau \in \Omega_{m}$

6) For each $m \geq m^{\prime}$, check if $\Omega_{m+1}=\Omega_{m}$ and denote by $\Omega^{*}=\Omega_{m^{*}}$, where $m^{*}$ is the smallest integer such that $m^{*} \geq m^{\prime}$ and $\Omega_{m^{*}+1}=\Omega_{m^{*}}$ (note that $m^{*} \leq n+2$ ).

7) If the gradient of a given state component $\left(x_{j}, j=\right.$ $1, \cdots, n$ ) belongs to $\Omega^{*}$ (namely if $\nabla x_{j} \in \Omega^{*}$ ) on a given neighbourhood of $x_{0}$, then $x_{j}$ is weakly locally observable at $x_{0}$. If this holds for all the state components, the state $x$ is weakly locally observable at $x_{0}$. Finally, if the dimension of $\Omega^{*}$ is smaller than $n$ on a given neighbourhood of $x_{0}$, then the state is not weakly locally observable at $x_{0}$.

\section{ROBOTIC APPLICATION}

\section{A. The system}

We consider a vehicle that moves in a $3 D$-environment. We assume that the dynamics of the vehicle are affected by the presence of a disturbance (e.g., this could be an aerial vehicle in presence of wind). We assume that the direction of the disturbance is constant in time and a priori known. Conversely, the disturbance magnitude is unknown and time dependent. The vehicle is equipped with speed sensors (e.g., airspeed sensors in the case of an aerial vehicle), gyroscopes and a bearing sensor (e.g., monocular camera). We assume that all the sensors share the same frame (in other words, they are extrinsically calibrated). Without loss of generality, we define the vehicle local frame as this common frame. The airspeed sensors measure the vehicle speed with respect to the air in the local frame. The gyroscopes provide the angular speed in the local frame. Finally, the bearing sensor provides the bearing angles of the features in the environment expressed in its own local frame. We consider the extreme case of a single point feature and, without loss of generality, we set the origin of the global frame at this point feature (see figure 1 for an illustration). Additionally, we assume that the $z$-axis of the global frame is aligned with the direction of the disturbance.

Our system can be characterized by the following state:

$$
X \triangleq\left[x, y, z, q_{t}, q_{x}, q_{y}, q_{z}\right]^{T}
$$

where $r=[x, y, z]$ is the position of the vehicle in the global frame and $q=q_{t}+q_{x} i+q_{y} j+q_{z} k$ is the unit quaternion that describes the transformation change between the global and the local frame. The dynamics are

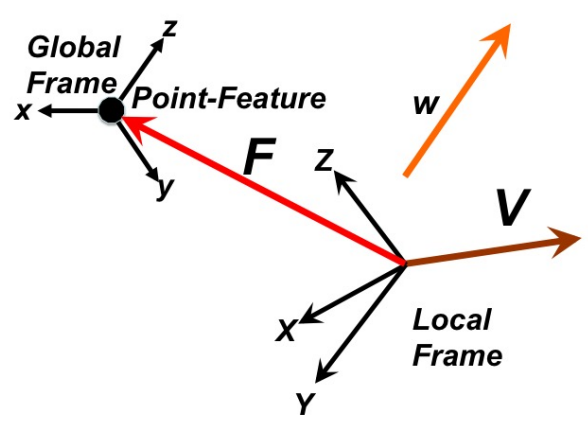

Fig. 1: Local and global frame for the considered problem. The $z$-axis of the latter is aligned with the direction of the disturbance (assumed to be known and constant in time). The speed $V$ is the vehicle speed with respect to the air, which differs from the ground speed because of the disturbance $(w)$.

affected by the presence of the disturbance. The disturbance is characterized by the following vector (in the global frame):

$$
\bar{w}=w\left[\begin{array}{l}
0 \\
0 \\
1
\end{array}\right]
$$

where $w$ is its unknown magnitude.

In the sequel, for each vector defined in the $3 D$ space, the pedix $q$ will be adopted to denote the corresponding imaginary quaternion. For instance, regarding the vehicle position, we have: $r_{q}=0+x i+y j+z k$. Additionally, we denote by $V$ and $\Omega$ the following physical quantities:

- $V=\left[V_{x}, V_{y}, V_{z}\right]$ is the vehicle speed with respect to the air expressed in the local frame (hence, $w k+q V_{q} q^{*}$ is the vehicle speed with respect to the ground expressed in the global frame).

- $\Omega \triangleq\left[\Omega_{x} \Omega_{y} \Omega_{z}\right]$ is the angular speed (and $\Omega_{q}=0+$ $\left.\Omega_{x} i+\Omega_{y} j+\Omega_{z} k\right)$.

The dynamics of the state are:

$$
\left[\begin{array}{ll}
\dot{r}_{q} & =w k+q V_{q} q^{*} \\
\dot{q} & =\frac{1}{2} q \Omega_{q}
\end{array}\right.
$$

The monocular camera provides the position of the feature in the local frame $\left(F_{q}=-q^{*} r_{q} q\right)$ up to a scale. Hence, it provides the ratios of the components of $F$ :

$$
h_{c a m}(X) \triangleq\left[h_{u}, h_{v}\right]^{T}=\left[\frac{\left(q^{*} r_{q} q\right)_{x}}{\left(q^{*} r_{q} q\right)_{z}}, \frac{\left(q^{*} r_{q} q\right)_{y}}{\left(q^{*} r_{q} q\right)_{z}}\right]^{T}
$$

where the pedices $x, y$ and $z$ indicate respectively the $i, j$ and $k$ component of the corresponding quaternion. We have also to consider the constraint $q^{*} q=1$. This provides the further observation:

$$
h_{\text {const }}(X) \triangleq q^{*} q
$$


Our system is characterized by the state in (4), the dynamics in (6) and the three outputs $h_{u}, h_{v}$ and $h_{\text {const }}$ in (7) and (8).

\section{B. Observability in absence of disturbance}

Our system is characterized by the state in (4), the dynamics in (6) with $w=0$ and the three outputs $h_{u}, h_{v}$ and $h_{\text {const }}$ in (7) and (8).

By comparing (6) with (1) we obtain that our system is characterized by six known inputs $\left(m_{u}=6\right)$ that are: $u_{1}=$ $\Omega_{x}, u_{2}=\Omega_{y}, u_{3}=\Omega_{z}, u_{4}=V_{x}, u_{5}=V_{y}$ and $u_{6}=V_{z}$. Additionally, we obtain:

$f^{1}=\frac{1}{2}\left[\begin{array}{c}0 \\ 0 \\ 0 \\ -q_{x} \\ q_{t} \\ q_{z} \\ -q_{y}\end{array}\right], f^{2}=\frac{1}{2}\left[\begin{array}{c}0 \\ 0 \\ 0 \\ -q_{y} \\ -q_{z} \\ q_{t} \\ q_{x}\end{array}\right], f^{3}=\frac{1}{2}\left[\begin{array}{c}0 \\ 0 \\ 0 \\ -q_{z} \\ q_{y} \\ -q_{x} \\ q_{t}\end{array}\right]$

$f^{4}=\left[\begin{array}{c}q_{t}^{2}+q_{x}^{2}-q_{y}^{2}-q_{z}^{2} \\ 2 q_{t} q_{z}+2 q_{x} q_{y} \\ 2 q_{x} q_{z}-2 q_{t} q_{y} \\ 0 \\ 0 \\ 0 \\ 0\end{array}\right], f^{5}=\left[\begin{array}{c}2 q_{x} q_{y}-2 q_{t} q_{z} \\ q_{t}^{2}-q_{x}^{2}+q_{y}^{2}-q_{z}^{2} \\ 2 q_{t} q_{x}+2 q_{y} q_{z} \\ 0 \\ 0 \\ 0 \\ 0 \\ f^{6}=\left[\begin{array}{c}2 q_{t} q_{y}+2 q_{x} q_{z} \\ 2 q_{y} q_{z}-2 q_{t} q_{x} \\ q_{t}^{2}-q_{x}^{2}-q_{y}^{2}+q_{z}^{2} \\ 0 \\ 0 \\ 0\end{array}\right]\end{array}\right]$

Finally, in absence of disturbance we have:

$$
g=\left[\begin{array}{llllll}
0,0,0,0,0,0 & 0
\end{array}\right]^{T}
$$

In this case we can apply the observability rank condition, i.e., algorithm 1 to obtain the observable codistribution. We compute the codistribution $\Omega_{0}$ by computing the gradients of the three functions $h_{u}, h_{v}$ and $h_{\text {const }}$. We obtain that this codistribution has dimension equal to 3 . We use algorithm 1 to compute $\Omega_{1}$. We obtain that its dimension is 4 . In particular, the additional covector is obtained by the gradient of the following Lie derivative:

$$
\mathcal{L}_{f^{4}} h_{u}
$$

In other words:

$$
\Omega_{1}=\operatorname{span}\left\{\nabla h_{u}, \nabla h_{v}, \nabla h_{\text {const }}, \nabla \mathcal{L}_{f^{4}} h_{u},\right\}
$$

All the remaining first order Lie derivatives have gradient that is in the above codistribution. Additionally, by an explicit computation, it is easy to realize that $\Omega_{2}=\Omega_{1}$. This means that algorithm 1 has converged and the observable codistribution is $\Omega_{1}$.

By an explicit computation, it is possible to check that the gradients of the components of the vector $F$ belong to $\Omega_{1}$. This means that all the observable modes are the components of $F$, i.e., the position of the feature in the local frame (obviously, the fourth observable mode is the norm of the quaternion). In particular, no component of the vehicle orientation is observable.

\section{Observability in presence of the disturbance}

We now consider the case when the dynamics are affected by the presence of the disturbance. By comparing (6) with (1) we obtain that the vector fields that characterize the dynamics are the same that characterize the dynamics in absence of disturbance with the exception of the last one, which becomes:

$$
g=[0,0,1,0,0,0,0]^{T}
$$

To derive the observability properties we apply the proposed analytic tool, by following the seven steps provided in section II-C. We have:

\section{First Step}

We start by computing the Lie derivatives of the outputs $h_{u}$, $h_{v}$ and $h_{\text {const }}$ along the vector field $g$. We find that the result differs from zero for the first two outputs. Hence, we use the first output $\left(h_{u}\right)$ to define $L_{g}^{1}$ (we could choose also the second output $\left.h_{v}\right)$. In particular, we obtain: $L_{g}^{1} \triangleq \mathcal{L}_{g} h_{u}=$ $\left\{-y\left(2 q_{t} q_{z}-2 q_{x} q_{y}\right)-x\left(q_{t}^{2}-q_{x}^{2}+q_{y}^{2}-q_{z}^{2}\right)\right\} /\left\{\left[z\left(q_{t}^{2}-q_{x}^{2}-\right.\right.\right.$ $\left.\left.\left.q_{y}^{2}+q_{z}^{2}\right)+2 x\left(q_{t} q_{y}+q_{x} q_{z}\right)+2 y\left(q_{y} q_{z}-q_{t} q_{x}\right)\right]^{2}\right\}$

\section{Second Step}

We compute the codistribution $\Omega_{0}$ by computing the gradients of the three functions $h_{u}, h_{v}$ and $h_{\text {const. }}$. This coincides with the case without disturbance, and we obtain that this codistribution has dimension equal to 3 .

We use algorithm 2 to compute $\Omega_{1}$. We obtain that its dimension is 5 . In particular, the additional two independent covectors are obtained by the gradients of the following two Lie derivatives:

$$
\mathcal{L}_{f^{4}} h_{u}, \quad \mathcal{L}_{\frac{g}{L_{g}^{1}}} h_{v}
$$

In other words:

$$
\Omega_{1}=\operatorname{span}\left\{\nabla h_{u}, \nabla h_{v}, \nabla h_{\text {const }}, \nabla \mathcal{L}_{f^{4}} h_{u}, \nabla \mathcal{L}_{\frac{g}{L_{g}^{1}}} h_{v}\right\}
$$

All the remaining first order Lie derivatives have gradient that is in the above codistribution.

\section{Third Step}

We compute ${ }^{1} \phi_{1},{ }^{2} \phi_{1},{ }^{3} \phi_{1},{ }^{4} \phi_{1},{ }^{5} \phi_{1}$ and ${ }^{6} \phi_{1}$ by using algorithm 3 . We obtain that all these vectors vanish. As a result, all the subsequent steps of algorithm 3 provide null vectors. Therefore, the assumptions of Lemma 1 are trivially met. We set $m^{\prime}=0$ and we skip to the sixth step.

\section{Sixth Step}


We use algorithm 2 to compute $\Omega_{2}$ and we obtain:

$$
\Omega_{2}=\Omega_{1}+\operatorname{span}\left\{\nabla \mathcal{L}_{f^{4}} \mathcal{L}_{\frac{g}{L_{g}^{1}}} h_{v}\right\}
$$

Hence, its dimension is 6 . Finally, by using again algorithm 2 it is possible to compute $\Omega_{3}$ and to check that $\Omega_{3}=\Omega_{2}$. This means that the algorithm has converged and the observable codistribution is $\Omega^{*}=\Omega_{2}$.

\section{Seventh Step}

By computing the distribution orthogonal to the codistribution $\Omega^{*}$ we can find the continuous symmetry that characterizes the unobservable space [20]. By an explicit computation we obtain the following vector:

$$
\left[-y, x, 0,-\frac{q_{z}}{2},-\frac{q_{y}}{2}, \frac{q_{x}}{2}, \frac{q_{t}}{2}\right]^{T}
$$

This symmetry corresponds to an invariance with respect to a rotation around the $z$-axis of the global frame. This means that we have a single unobservable mode that is the yaw in the global frame We conclude by remarking that the presence of the disturbance, even if its magnitude is unknown and is not constant, makes observable the roll and the pitch angles. This result is similar to the result that we obtain in the case of visual and inertial sensor fusion in presence of gravity. The presence of gravity makes observable the roll and the pitch angles, even if its magnitude is unknown [21]. What it is non intuitive in the case now investigated, is that, not only the magnitude of the disturbance is unknown, but it is also time dependent.

\section{Simulations}

\section{Simulated trajectories and robot sensors}

The trajectories are simulated as follows. The equations in (6) are discretized with a time step of $0.01 \mathrm{~s}$. Each trial lasts $200 \mathrm{~s}$. The initial vehicle speed is set to zero. The initial position is set equal to $\left[\begin{array}{lll}0.5 & 0.5 & 0.5\end{array}\right] \mathrm{m}$. The vehicle motion is randomly generated. The angular speed, i.e. $\Omega$, is Gaussian. Specifically, its value at each step follows a zero mean Gaussian distribution with covariance matrix equal to $(1 \mathrm{deg})^{2} I_{3}$, where $I_{3}$ is the identity $3 \times 3$ matrix. At each time step, the vehicle speed is incremented by adding a random vector with zero mean Gaussian distribution. In particular, the covariance matrix of this distribution is set equal to $\sigma^{2} I_{3}$, with $\sigma=10^{-4} \mathrm{~m}$. Finally, we simulate the disturbance as a vector along the $z$-axis, whose magnitude is generated as a random Gaussian variable, with mean value $0.2 \mathrm{~ms}^{-1}$ and variance $0.05^{2} \mathrm{~m}^{2} \mathrm{~s}^{-2}$. Typical trajectories, obtained with this setting, are displayed in figures 2 and 3 .

The vehicle is equipped with proprioceptive sensors able to measure at each time step the speed with respect to the air and the angular speed. These measurements are affected by errors. Specifically, each measurement is generated at every time step of $0.01 s$ by adding to the true value a random error that follows a Gaussian distribution. The mean value of this

\footnotetext{
${ }^{6}$ Note that the chosen global frame is aligned with he direction of the disturbance (fig. 1). Hence, what is unobservable is a rotation around the direction of the disturbance.
}

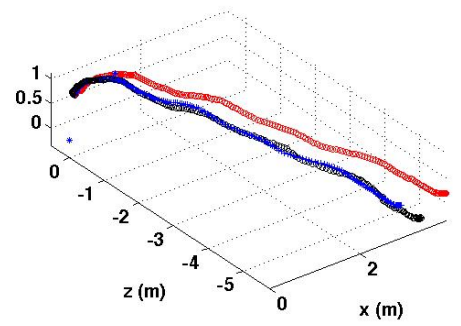

Fig. 2: A typical simulated trajectory in absence of disturbance. In blue the true trajectory, in red the trajectory estimated by only integrating the gyroscope and the airspeed measurements and in black the trajectory estimated by the proposed EKF.

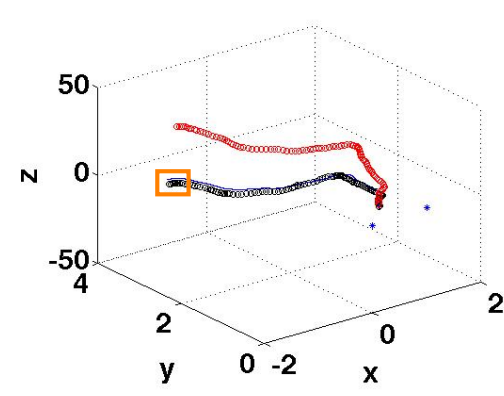

(a)

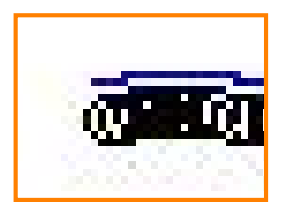

Fig. 3: A typical simulated trajectory in presence of disturbance along the $z$-axis. In blue the true trajectory, in red the trajectory estimated by only integrating the gyroscope and the airspeed measurements and in black the trajectory estimated by the proposed EKF.

error is zero and the standard deviation is $0.01 \mathrm{~ms}^{-1}$ for the airspeed and $1 \mathrm{deg} s^{-1}$ for the gyroscope. Regarding the exteroceptive measurements, they are generated at a lower frequency. Specifically, the measurements are generated each $0.1 s$. Also these measurements are affected by errors. Specifically, each measurement is generated by adding to the true value a random error that follows a zero mean Gaussian distribution, with variance $1 \mathrm{deg}^{2}$. We simulate two point features, one at the origin and the other one at the position $\left[\begin{array}{lll}1 & 0 & 1\end{array}\right] \mathrm{m}$ (note that the result of the observability analysis is independent of the number of point features).

\section{Estimation results}

We adopt an EKF that estimates an extended state that includes the state in 4 and the unknown input $(w)$ together with its first order time derivative $\left(w^{(1)} \triangleq \frac{d w}{d t}\right)$. In other words, the state is: $\left[x, y, z, q_{t}, q_{x}, q_{y}, q_{z}, w, w^{(1)}\right]^{T}$. Its dynamics are given in (6) with the two further equations $\dot{w}=w^{(1)}$ and $\dot{w}^{(1)}=w^{(2)}$. In order to implement the prediction phase of our EKF we have to provide the value of $w^{(2)}\left(\triangleq \frac{d^{2} w}{d t^{2}}\right)$, which is unknown. We set this quantity to zero. Note that the simulated trajectory does not satisfy this hypothesis since the disturbance is randomly generated. 
However, the estimator is able to provide good performance as it is shown in figures $4 / 7$ Figure 2 displays the estimated trajectory in absence of the disturbance. The red line is the trajectory obtained by only using the proprioceptive measurements, while the black line is the trajectory obtained by the proposed EKF. The true trajectory is in blue. Both the estimated trajectories diverge. However, the divergence is slower for the trajectory estimated by the EKF. This divergence is consistent with our observability analysis. The vehicle position is not observable (both with and without disturbance). Figure 3 displays the same of figure 2 for a trial in presence of the disturbance. Note that, in this case, the $z$-axis has now a different scale (much larger). This is to show the trajectory obtained by only using the proprioceptive measurements, whose divergence is much faster due to the presence of the disturbance along the $z$-axis. In the remaining figures, we show the performance of the proposed EKF in estimating the distances of the two features and the roll, the pitch and the yaw angles. In accordance with our observability analysis, the distances of the features are observable both with and without the disturbance. In contrast, the roll and the pitch angles are observable only in presence of the disturbance. Finally, the yaw angle is always unobservable. All these results are clearly confirmed by our simulation results.
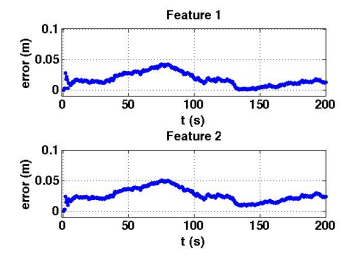
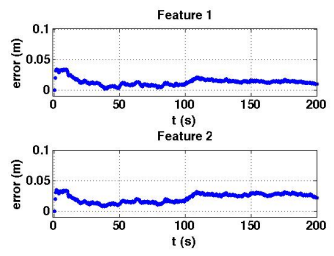

Fig. 4: Estimation error on the distance of the two observed point features with and without disturbance (right and left side, respectively).
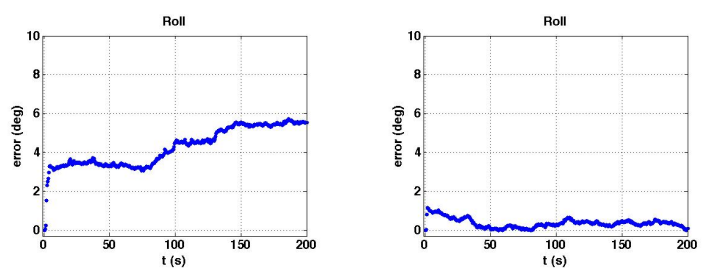

Fig. 5: As in fig. 4 but for the roll angle.
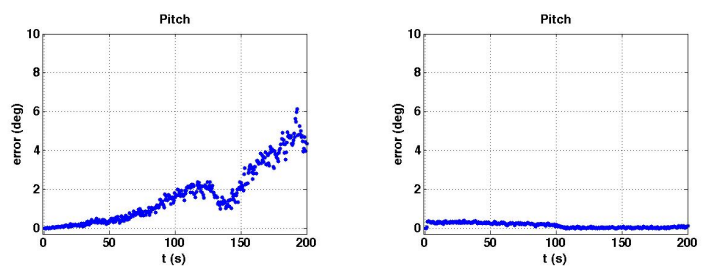

Fig. 6: As in fig. 4 but for the pitch angle.
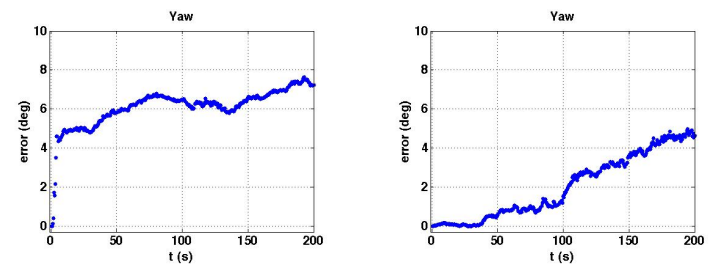

Fig. 7: As in fig. 5 but for the yaw angle.

Figure 4 shows the error on the two distances, in absence and in presence of the disturbance, respectively. We note that, the error does not increase with time in both cases. Figures 5 , 6 and 7 show the error on the roll, pitch and yaw angles, respectively. The left side is in absence of the disturbance while the right side is in presence of the disturbance. In absence of the disturbance, the error increases with time for all the three angles. In presence of the disturbance, it does not increase for the roll and the pitch angle.

We conclude this section by remarking that our simulations fully agree with the observability analysis provided in section III-C Also note that we showed the result of a single trial. However, we found similar results by running many trials.

\section{CONCLUSION}

In this paper we provided the analytic criterion to perform the observability analysis of a system in presence of disturbances together with an application of this criterion in order to obtain the observability properties of a robotic system. The analytic criterion is the solution of a fundamental open problem in nonlinear observability/estimation theory, which is the nonlinear unknown input observability problem. The first part of the paper provided this solution in the case of nonlinear systems driven by a single unknown input and multiple known inputs. In the second part of the paper we analyzed the observability properties of a system that consists of a robot that moves in $3 D$ and when its dynamics are affected by an external disturbance (e.g., due to the presence of wind). For this robotic system the analytic criterion provided automatically the observable states. To corroborate the results of this observability analysis, we performed extensive simulations. We showed that, a simple estimator based on an Extended Kalman Filter, provides results that fully agree with what we could expect from the observability analysis.

Note that more robotic applications of our analytic solution can be found in [24]. In particular, in section 5.1, we analyze the observability of the well known unicycle when one of the two inputs (which are the linear and the angular speed) is unknown. When the linear speed is unknown and when the observations consist of angle measurements (e.g., the ones provided by a monocular camera), all the measurements only consist of angle measurements (the angular speed and the camera measurements). In this case, it is obvious that, with respect to the case when the linear speed is a known input, the observability space loses one degree, which corresponds to the absolute scale. It is amazing that, our simple analytic solution, automatically detects this unobservable direction 
(see more details in section 5.1 in [24]).

Finally, in this paper we provided the analytic solution of the UIO problem for driftless systems and in the case of a single unknown input. Recently, the general case that includes a nonlinear drift and multiple unknown inputs was investigated and the analytic criterion to check the state observability of these more general systems has been derived [25]. To this regard, we remark two important aspects:

1) The derivation of the analytic condition in the general case builds on the solution provided in this paper. The main difference comes from the fact that many scalar quantities become tensors (e.g., $L_{g}^{1}$ becomes a two index tensor of type $(1,1)$; the scalar $\tau$ in 3 becomes a three index tensor of type $(2,1)$ ).

2) In contrast with the surprising simplicity and compact architecture of the general solution (see sections 3.3 and 4.2 in [25]), its derivation is very complex. Specifically, it is based on a subtle analogy with the theory of General Relativity and, consequently, on the use of Ricci calculus. In this analogy, the presence of the drift term corresponds to the presence of a time dimension in relativity and the presence of unknown inputs corresponds to the space dimension in relativity (i.e., in the aforementioned analogy, the space dimension of relativity equals the number of unknown inputs). In this sense, the case analyzed in this paper corresponds to the trivial case of a space-time frozen with respect to time and with a single spatial dimension (this is the reason why the derivations of the results provided in this paper (available in [24]) do not require the use of Ricci calculus).

The general solution provided in [25] allows us to automatically derive the observability properties of any nonlinear system driven by disturbances. Note that, in sections $5.4-5.7$ of [25], we revisit the visual-inertial sensor fusion problem. The results include the ones previously obtained [21], [22]. However, with respect to the former derivation, the new one was obtained automatically and the results are more general.

\section{REFERENCES}

[1] G. Basile and G. Marro. On the observability of linear, time invariant systems with unknown inputs. J. Opt. Theory Appl., 3:410-415, 1969.

[2] F. A. W. Belo, P. Salaris, and A. Bicchi, 3 Known Landmarks are Enough for Solving Planar Bearing SLAM and Fully Reconstruct Unknown Inputs, IROS 2010, Taipei, Taiwan

[3] S.P. Bhattacharyya Observer design for linear systems with unknown inputs IEEE Trans. on Aut. Control, 23 (1978)

[4] M. Bryson and S. Sukkarieh, Observability Analysis and Active Control for Airbone SLAM, IEEE Transaction on Aerospace and Electronic Systems, vol. 44, no. 1, 261-280, 2008

[5] A. Censi, A. Franchi, L. Marchionni, and G. Oriolo. Simultaneous calibration of odometry and sensor parameters for mobile robots. IEEE Transactions on Robotics, 29(2):475?492, April 2013.

[6] R. Guidorzi and G. Marro. On Wonham stabilizability condition in the synthesis of observers for unknown-input systems. Automatic Control, IEEE Transactions on, 16(5):499-500, oct 1971.

[7] Chao X. Guo and Stergios I. Roumeliotis, IMU-RGBD Camera 3D Pose Estimation and Extrinsic Calibration: Observability Analysis and Consistency Improvement, ICRA 2013, Karlsruhe, Germany

[8] Hermann R. and Krener A.J., 1977, Nonlinear Controllability and Observability, Transaction On Automatic Control, AC-22(5): 728-740
[9] J. A. Hesch, D. G. Kottas, S. L. Bowman, and S. I. Roumeliotis, Towards consistent vision-aided inertial navigation, Int. Workshop on the Algorithmic Foundations of Robotics, Cambridge, MA, Jun, 2012.

[10] J.A. Hesch, D.G. Kottas, S.L. Bowman, and S.I. Roumeliotis, "Camera-IMU-based Localization: Observability Analysis and Consistency Improvement," International Journal of Robotics Research, 33(1), Jan. 2014, pp. 182-201.

[11] G. Huang, A.I. Mourikis, S.I. Roumeliotis: "Observability-based Rules for Designing Consistent EKF SLAM Estimators,' International Journal of Robotics Research, 29(5), pp. 502-528, 2010.

[12] G. Huang, A.I. Mourikis, S.I. Roumeliotis: "A Quadratic-Complexity Observability-Constrained Unscented Kalman Filter for SLAM," IEEE Transactions on Robotics, 29(5), pp. 1226-1243, 2013.

[13] G. Huang, M. Kaess, J. Leonard, Towards consistent visual-inertial navigation, IEEE Int. Conf. on Rob. and Aut. 2014

[14] Isidori A, Nonlinear Control Systems, 3ed., London, Spring Verlag 1995.

[15] E. Jones and S. Soatto, "Visual-inertial navigation, mapping and localization: A scalable real-time causal approach", The International Journal of Robotics Research, vol. 30, no. 4, pp. 407-430, Apr. 2011.

[16] J. Kelly and G. Sukhatme, Visual-inertial simultaneous localization, mapping and sensor-to-sensor self-calibration, Int. Journal of Robotics Research, vol. 30, no. 1, pp. 56-79, 2011.

[17] D. G. Kottas, J. A. Hesch, S. L. Bowman, and S. I. Roumeliotis, On the consistency of vision-aided inertial navigation, in Proc. of the Int. Symposium on Experimental Robotics, Canada, Jun 2012.

[18] Kwang Wee Lee, Wijesoma, W.S. and Javier, I.G., On the Observability and Observability Analysis of SLAM, IEEE/RSJ International Conference on Intelligent Robots and Systems, 9-15 Oct. 2006, Beijing, China.

[19] M. Li and A. I. Mourikis, Improving the accuracy of EKF-based visualinertial odometry, ICRA 2012, St. Paul, MN.

[20] A. Martinelli, State Estimation Based on the Concept of Continuous Symmetry and Observability Analysis: the Case of Calibration, IEEE Transactions on Robotics, vol. 27, no. 2, pp. 239-255, 2011

[21] A. Martinelli, Observability Properties and Deterministic Algorithms in Visual-Inertial Structure from Motion, Found. and Trends in Robotics, Vol 3, 139-209.

[22] A. Martinelli, Minimalistic sensor design in visual-inertial structure from motion, 2015 IEEE/RSJ International Conference on Robotics and Automation, Seattle (USA).

[23] A. Martinelli, Nonlinear Unknown Input Observability: Analytical expression of the observable codistribution in the case of a single unknown input, SIAM - CT15, 2015, Paris, France.

[24] A. Martinelli. Nonlinear Unknown Input Observability: The Analytic Solution in the case of a Single Unknown Input., arXiv:1501.02968 [math.OC]

[25] A. Martinelli. Nonlinear Unknown Input Observability: The General Analytic Solution., arXiv:1704.03252 [math.OC]

[26] Mirzaei F.M. and Roumeliotis S.I., A Kalman filter-based algorithm for IMU-camera calibration: Observability analysis and performance evaluation, Transactions on Robotics, 2008, Vol. 24, No. 5, 1143-1156

[27] F.M. Mirzaei, D.G. Kottas, and S.I. Roumeliotis, 3D Lidar-Camera Intrinsic and Extrinsic Calibration: Observability Analysis and Analytical Least Squares-based Initialization, International Journal of Robotics Research, 31(4), Apr. 2012, pp. 452-467.

[28] G. Panahandeh, C. X. Guo, M. Jansson, and S. I. Roumeliotis, "Observability Analysis of a Vision-Aided Inertial Navigation System using Planar Features on the Ground", in Proc. of the IEEE/RSJ International Conference on Intelligent Robots and Systems (IROS), pp. 4187-4194, Tokyo, Japan, Nov. 3-7, 2013.

[29] Perera, L.D.L., Melkumyan, A. and Nettleton, E., On the linear and nonlinear observability analysis of the SLAM problem, IEEE International Conference on Mechatronics, 14-17 April 2009, Malaga.

[30] N. Trawny, G. Huang, A.I. Mourikis, S.I. Roumeliotis: "Observabilitybased Consistent EKF Estimators for Multi-robot Cooperative Localization," Autonomous Robots, 30(1), pp. 99-122, 2011.

[31] Vidal-Calleja T., Bryson M., Sukkarieh S., Sanfeliu A., Andrade-Cetto J., On the Observability of Bearing-only SLAM, EEE International Conference on Robotics and Automation, 10-14 April 2007, Roma.

[32] S.H. Wang, E.J. Davison, P. Dorato Observing the states of systems with unmeasurable disturbance IEEE Trans. on Aut. Control, 20 (1975)

[33] Wang Z. and Dissanayake, G., Observability analysis of SLAM using fisher information matrix, International Conference on Control, Automation, Robotics and Vision, 2008, Hanoi. 